\title{
Modelagem da distribuição diamétrica para Eucalipto seminal na região Centro Oeste de Mato Grosso
}

\author{
Martha Aguiar dos Santos ${ }^{1}$ João Paulo Sardo Madi ${ }^{1}$ Ronaldo Drescher ${ }^{1}$ Rômulo Môra ${ }^{1}$ Scheila Cristina \\ Biazatti $^{1}$
}

${ }^{1}$ Universidade Federal de Mato Grosso, Faculdade de Engenharia Florestal, Av. Fernando Correa da Costa, 2367, Cuiabá-MT, 78060-900

*Author for correspondence: marthaguiar@gmail.com

Received: December 2018 / Accepted: March 2019 / Published: June 2019

\begin{abstract}
Resumo
O presente trabalho teve como objetivo avaliar o desempenho de diferentes funções de densidade probabilísticas (FDP) na caracterização da estrutura diamétrica de um povoamento híbrido de Eucalytpus urophylla $x$ Eucalyptus grandis de doze anos, no município de Tangará da Serra, Mato Grosso. Foram mensurados os diâmetros à altura do peito dos indivíduos amostrados e agrupados em seis classes, com amplitude de $4,5 \mathrm{~cm}$. Foram calculadas as estatísticas descritivas e verificado o comportamento da variável diâmetro à altura do peito DAP). Para avaliar a distribuição diamétrica dessa espécie foram ajustadas funções densidade de probabilidade: Normal, Log-Normal, Weibull-2P, Gamma e Weibull-3P. A qualidade dos ajustes foi verificada através do teste de aderência de KolmogorovSmirnov e Critério de Informação de Akaike (AIC). A função que melhor estimou a distribuição diamétrica foi a Weibull-3P, com menor valor para o teste de aderência de Kolmogorov-Smirnov e menor AIC.

Palavras-chave: Classe diamétrica, Modelo probabilístico, Weibull.
\end{abstract}

\begin{abstract}
The present work had as objective to evaluate the performance of different probabilistic density functions (PDF) in the diametric structure characterization of a hybrid stand of Eucalyptus urophylla $x$ Eucalyptus grandis of twelve years old in the municipality of Tangará da Serra, Mato Grosso. The diameters were measured at the breast height of the individuals sampled and grouped into six classes, with an amplitude of 4.5 $\mathrm{cm}$. The descriptive statistics were calculated and the behavior of the variable diameter at breast height was verified. To evaluate the diametric distribution of this species were adjusted probability density functions: Normal, Log-Normal, Weibull-2P, Gamma e Weibull-3P, and the adjustment quality was verified by the Kolmogorov-Smirnov test and the Akaike Information Criterion (AIC). The function that best estimated the diametric distribution was Weibull-3P, with lower value for the Kolmogorov-Smirnov adhesion test and lower AIC.
\end{abstract}

Keywords: Diametric class, Probabilistic model, Weibull function.

\section{Introdução}

O setor florestal brasileiro, nos últimos anos, tornouse um dos mais relevantes no cenário mundial. Com uma área de 7,84 milhões de hectares de reflorestamento, os plantios de floresta são responsáveis por $91 \%$ de toda a madeira produzida para fins industriais, destacando-se como segmento de maior potencial de contribuição para a construção de uma economia verde (IBÁ, 2017).
Dentre as espécies plantadas de maior importância, tem-se as do gênero Eucalyptus, que tiveram o maior aumento de áreas cultivadas e representam mais de 70\% do total de plantios florestais (IBÁ 2017). Segundo Longue Júnior e Colodette (2013), dentre os fatores que conferem destaque aos eucaliptos brasileiros, pode-se citar a alta produtividade das florestas atribuída ao clima tropical ou subtropical na maioria do território, o que permite um crescimento ininterrupto e, consequentemente, um rápido acúmulo de biomassa.

Nesse sentido, torna-se fundamental para as empresas florestais a quantificação e a prognose, com confiabilidade, do estoque de suas florestas. Sendo a quantificação de crescimento e produção, segundo Eisfeld et al. (2005), uma condição essencial para definir a utilização da floresta, além de fornecer informações que subsidiam a tomada de decisões para a maioria das atividades ligadas a toda cadeia produtiva de florestas plantadas.

A distribuição diamétrica é a ferramenta simples e eficiente para caracterizar a estrutura de uma floresta (Arce 2004). Considerando a frequência por classes de diâmetros e podendo, eventualmente, incluir a área basal, o volume, e os incrementos em diâmetro, em área basal, em volume e em valor econômico, como variáveis agrupadas por classes de diâmetros. Os modelos de distribuição diamétrica têm sido bastante utilizados, tanto em plantios florestais como em florestas nativas, pois fornecem informações detalhadas quanto à estrutura das florestas.

Uma importante ferramenta de apoio à tomada de decisão nos povoamentos florestais, são as funções densidade de probabilidade (FDP). Essas distribuições permitem obter a probabilidade de ocorrência das árvores dentro de intervalos diamétricos previamente delimitados. Além disso, permite distinguir diferentes tipos florestais e de viabilizar a prognose dos múltiplos produtos (Scolforo 2006).

A representação da distribuição diamétrica por meio de funções de densidade probabilística, com baixos níveis de erro de estimativa, possui um grande valor para empresas e profissionais que trabalham no planejamento e manejo florestal, tendo em vista que, quanto mais se conhece sobre a floresta, melhor podem-se planejar as atividades de industrialização e beneficiamento da madeira, bem como otimizar os investimentos e a lucratividade dos povoamentos florestais (Téo et al. 2012).

Sendo assim, o objetivo deste estudo foi avaliar o desempenho de funções de densidade probabilísticas para representar a estrutura diamétrica um povoamento de híbrido de Eucalytpus urophylla x Eucalyptus grandis no município de Tangará da Serra, Mato Grosso. 


\section{Material e Métodos}

O estudo foi realizado em um povoamento híbrido de Eucalytpus urophylla $x$ Eucalyptus grandis conduzido para serraria, com idade de 12 anos, estabelecido sob espaçamento $4 \mathrm{~m}$ x 2,20 m e área total de 140,38 hectares, pertencente a Fazenda São João, nas coordenadas $57^{\circ} 55^{\prime} 38^{\prime \prime O}$ e $14^{\circ} 28^{\prime} 4^{\prime \prime}$ 'S, no município de Tangará da Serra, Mato Grosso. No povoamento foram realizadas desramas nas árvores selecionadas para futuro corte para serraria e os desbastes foram realizados primeiro aos 3,5 anos de idade e o segundo aos 7 anos de idade.

O clima da região é do tipo Aw segundo classificação de Köppen (Alvares et al. 2013) e é classificado como tropical semiúmido com quatro a cinco meses secos e temperatura média mensal acima de $18{ }^{\circ} \mathrm{C}$, durante todo o ano (IBGE 2002).

Realizou-se o inventário florestal utilizando processo de amostragem sistemático, com o estabelecimento de 17 unidades de amostra circulares com 11,29 m de raio, totalizando 0,68 ha de área amostrada. Foram coletados o diâmetro à altura do peito (DAP) e altura total de 227 árvores. A partir da amostra, os dados foram agrupados por meio de análise prévia e critério empírico em seis classes diamétricas com intervalos de $4,5 \mathrm{~cm}$, procedimento realizado através do pacote fdth (Faria 2017) na linguagem $R$ (R Development Core Team 2017).

Para a estimativa da distribuição diamétrica do povoamento foram ajustadas as funções de densidade probabilísticas (FDP): Normal, Log-Normal, Weibull 2 parâmetros, Gamma e Weibull 3 parâmetros (Tabela 1). Todos os procedimentos foram implementados na linguagem R (R Development Core Team 2017) utilizando a função fitdistr do pacote MASS (Venables e Ripley 2002) que utiliza o método da máxima verossimilhança de distribuições univariadas.

Tabela 1. Funções de densidade probabilística (FDP) utilizadas no ajuste para um povoamento híbrido de Eucalytpus urophylla $x$ Eucalyptus grandis, aos 12 anos em Tangará da Serra, Mato Grosso.

\begin{tabular}{|c|c|c|}
\hline & Função & Condições \\
\hline Normal & $f(x)=\left(\frac{1}{\sigma \sqrt{2 \pi}}\right) e^{-\frac{1}{2}\left(\frac{x-\mu}{\sigma^{2}}\right)^{2}}$ & $\begin{array}{l}\sigma>0 \\
-\infty<x<+\infty \\
\infty<\mu<+\infty\end{array}$ \\
\hline $\begin{array}{c}\text { Log } \\
\text { normal }\end{array}$ & $f(x)=\left(\frac{1}{x\left(\sqrt{2 \pi \sigma^{2}}\right)}\right) e^{-\frac{1}{2} \frac{(\ln (x)-\mu)^{2}}{\sigma^{2}}}$ & $\begin{array}{l}x \geq a ; \sigma>0 \\
-\infty<x<+\infty \\
\infty<\mu<+\infty\end{array}$ \\
\hline Weibull 2P & $f(x)=\frac{c}{b}\left(\frac{x}{b}\right)^{c-1} e^{-\left(\frac{x}{b}\right)^{c}}$ & $\begin{array}{l}x \geq 0 \\
c, b>0\end{array}$ \\
\hline Weibull 3P & $f(x)=\frac{c}{b}\left(\frac{(x-a)}{b}\right)^{c-1} e^{-\left(\frac{x-a}{c}\right)^{c}}$ & $\begin{array}{l}x \geq 0 \\
c, b, a>0\end{array}$ \\
\hline Gamma & $f(x)=\frac{x^{\alpha-1} e^{\left(\frac{-x}{\beta}\right)}}{\beta^{\alpha} \Gamma(\alpha)}$ & $\begin{array}{l}x \geq a \\
-\infty<a<+\infty \\
\alpha, \beta>0\end{array}$ \\
\hline
\end{tabular}

Legenda: $\mathrm{f}(\mathrm{x})$ - função de densidade da variável x; $\mathrm{x}$ - diâmetro do centro de classe; $\mu$ - média aritmética; $\sigma$ - desvio padrão; $\pi$ - é a constante "pi" $(3,1416)$; $a$ - menor valor da variável observada; $b$ - parâmetro de escala; $\mathrm{c}$ - parâmetro de forma; $\Gamma$ é a função gama caracterizada pela seguinte expressão (para qualquer $\alpha \geq 0$ ): $\Gamma(\alpha)=\int_{0}^{\infty} u^{a-1} e^{-u} \delta$

$$
\begin{aligned}
& A s=\frac{1}{n} \sum\left[\frac{X i-\mu}{\sigma}\right]^{3} \\
& C=\frac{1}{n} \sum\left[\frac{X i-\mu}{\sigma}\right]^{4}-3
\end{aligned}
$$

Em que: $n=$ número de indivíduos; $X i=$ diâmetro à altura do peito de cada indivíduo; $\mu=$ média dos diâmetros à altura do peito; $\sigma=$ desvio padrão.

Para a seleção das funções de densidade probabilísticas mais aderentes foi realizado o teste de aderência de Kolmogorov-Smirnov (K-s) que testa a hipótese nula de que os diâmetros observados seguem as distribuições propostas pelas funções de densidade probabilísticas ajustadas ao nível de $5 \%$ de probabilidade conforme (Machado et al. 2010); (Téo et al. 2011); (Binoti et al. 2012).

Além disso, foi calculado o Critério de Informação de Akaike (AIC), teste que permite uma comparação entre modelos não aninhados e penalizam aqueles com maior número de parâmetros, adequando-os ao conceito de parcimônia. Quanto menor seu valor indica melhor ajuste (Wolfinger, 1993).

$$
A I C=-2 \ln (m v)+2 p
$$

Em que: mv: máxima verossimilhança e n: número de parâmetros do modelo.

\section{Resultados}

O conjunto de dados das 227 árvores de Eucalytpus urophylla $x$ Eucalyptus grandis apresentou variação na variável diâmetro à altura do peito entre $12,22 \mathrm{~cm}$ a 36,60 $\mathrm{cm}$, com amplitude de $24,38 \mathrm{~cm}$. A distribuição do número de árvores por classe apresentou tendência à distribuição normal com valores de média, mediana e moda próximos, sendo $24,24 \mathrm{~cm}, 24,44 \mathrm{~cm}$ e $24,7 \mathrm{~cm}$, 
respectivamente, influenciado pela presença de mais indivíduos nas classes 3 e 4, que representam os limites de 21 a $30 \mathrm{~cm}$ (Tabelas 2 e 3 ).

Tabela 2. Frequência observada de árvores nas classes diamétricas para um povoamento híbrido de Eucalytpus urophylla $x$ Eucalyptus grandis, aos 12 anos em Tangará da Serra, Mato Grosso

\begin{tabular}{cccccc}
\hline Cl & LI & LS & CC1 & FO & FR \\
\hline 1 & 12,0 & 16,5 & 14,25 & 13 & 5,73 \\
2 & 16,5 & 21,0 & 18,75 & 49 & 21,59 \\
3 & 21,0 & 25,5 & 21,25 & 66 & 29,07 \\
4 & 25,5 & 30,0 & 27,75 & 67 & 29,52 \\
5 & 30,0 & 34,5 & 30,25 & 29 & 12,78 \\
6 & 34,5 & 39,0 & 36,75 & 3 & 1,32 \\
\hline Total & & & & 227 & 100
\end{tabular}

Cl: Classe; LI: limite superior $(\mathrm{cm})$; LS: limite inferior $(\mathrm{cm})$; $\mathrm{CCl}$ : centro de classe $(\mathrm{cm})$; $\mathrm{FO}$ : frequência observada; FR: frequência relativa (\%).

Tabela 3. Estatísticas descritivas para DAP do povoamento do híbrido de Eucalytpus urophylla x Eucalyptus grandis, aos 12 anos em Tangará da Serra, Mato Grosso.

\begin{tabular}{cccc}
\hline \multicolumn{4}{c}{ Estatísticas descritivas - DAP (cm) } \\
\hline DAP médio & 24,24 & Ero Padrão & 0,324 \\
DAP mínimo & 36,60 & Desvio Padrão & 4,881 \\
DAP máximo & 12,22 & Variância & 23,8305 \\
No de indivíduos $^{\text {Mediana }}$ & 227 & Curtose & $-0,63240$ \\
Moda & 24,44 & Assimetria & $-0,15446$ \\
& 24,70 & Somatório & 55501,744 \\
\hline
\end{tabular}

A curva de distribuição diamétrica foi classificada como assimétrica negativa (assimetria à esquerda) devido o valor de média ser menor que a mediana e esta ser menor que a moda, seguindo essa ordem: $(\mu<\mathrm{Md}<\mathrm{Mo})$. O valor de curtose foi menor que 0,263 (Tabela 3), demonstrando que a distribuição apresenta uma curva leptocúrtica, ou seja, quando a distribuição é mais pontiaguda que a normal.

A Tabela 4 apresenta as funções de densidade probabilística testadas em ordem de maior aderência, seus parâmetros estimados, seus respectivos valores de AIC, assim como os valores de D estimado e calculados através do teste de Kolmogorov-Smirnov. Por meio desse teste, observou-se que os valores calculados foram menores que o tabelado, isto indica a aceitação da hipótese de nulidade, o que significa que as frequências esperadas e observadas são similares sob ponto de vista estatístico, ou seja, os modelos representaram com maior aderência o conjunto de dados.

Tabela 4. Parâmetros das funções de densidade probabilísticas ajustadas para um povoamento híbrido de Eucalytpus urophylla $x$ Eucalyptus grandis, aos 12 anos em Tangará da Serra, Mato Grosso.

\begin{tabular}{|c|c|c|c|c|}
\hline Função & Parâmetros & D cal & $D^{\prime}(95 \%)$ & AIC \\
\hline & $c=3,97$ & \multirow{3}{*}{$0,03163^{*}$} & \multirow{11}{*}{0,0903} & \multirow{3}{*}{1364,11} \\
\hline \multirow[t]{2}{*}{ Weibull 3P } & $b=18,72$ & & & \\
\hline & $a=7,30$ & & & \\
\hline \multirow{2}{*}{ Weibull $2 P$} & $c=5,70$ & $004143 *$ & & \multirow{2}{*}{1364,288} \\
\hline & $b=26,21$ & & & \\
\hline \multirow[t]{2}{*}{ Normal } & $\mu=24,24$ & $0,04658^{*}$ & & \multirow[t]{2}{*}{1367,006} \\
\hline & $\sigma=4,87$ & & & \\
\hline \multirow{2}{*}{ Gamma } & $\beta=23,16$ & $0.07408^{*}$ & & \multirow{2}{*}{1375,538} \\
\hline & $\alpha=0,95$ & & & \\
\hline Log & $\ln (\mu)=3,17$ & \multirow{2}{*}{$0,08806^{*}$} & & \multirow{2}{*}{1383,901} \\
\hline normal & $\ln (\sigma)=0,21$ & & & \\
\hline
\end{tabular}

Legenda: D' = D de Kolmogorov-Smirnov calculado; * Valor com significância no teste bilateral a 5\%; AIC: Critério de Informação de Akaike; $\mu=$ média aritmética; $\sigma=$ desvio padrão; $b, c, a, \alpha$ e $\beta=$ parâmetros estimados.

O melhor ajuste foi encontrado para a função Weibull 3P com AIC de 1364,111, seguido pela função Weibull 2P com AIC de 1364,289, portanto apresentando uma diferença de 0,2 unidades, assim consideram-se os modelos semelhantes. Pois, segundo Arnold (2010), quando a diferença no AIC for maior que 2 unidades, os modelos são considerados diferentes e o modelo com a menor AIC considerado o mais preciso.

A Log-normal foi a função de distribuição diamétrica que obteve o pior desempenho entre as testadas. A diferença nos valores de AIC entre esta função e a Weibull 3P foi de 19,8.

Para complementar a decisão de escolha da melhor função, para intervalos de classe de $4,5 \mathrm{~cm}$, foram traçadas as curvas de frequências estimadas sobre o histograma das frequências observadas (Figura 1). Essa análise gráfica permitiu ter uma maior clareza no julgamento da tendenciosidade dos modelos de distribuição diamétrica avaliados.

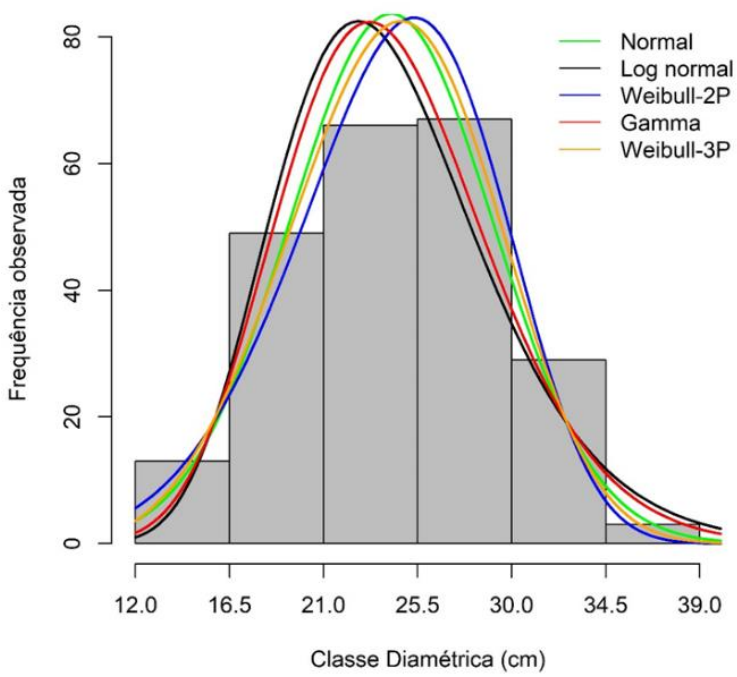

Figura 1. Distribuições de frequências observadas e estimadas pelas funções Normal, Log-Normal, Weibull 2 Parâmetros (2P), Gamma, Weibull 3 Parâmetros (3P) pelos centros de classes diamétrica, para um povoamento híbrido de Eucalytpus urophylla $x$ Eucalyptus grandis, aos 12 anos, em Tangará da Serra, Mato Grosso. 
As curvas estimadas pelos modelos se cruzam na quinta classe de diâmetro (Figura 1). A função Weibull $3 \mathrm{P}$ que apesar de ser selecionada como a melhor, apresentou tendência em subestimar as duas primeiras classes e últimas e superestimar as classes intermediárias, onde tem-se o maior número de árvores.

A função Weibull $2 \mathrm{P}$ apresentou praticamente $\mathrm{O}$ mesmo padrão, apenas na primeira classe não apresentou tendenciosidade.

A função Normal subestimou as duas primeiras classes, superestima na terceira classe, uma leve subestimativa na quarta classe, subestima na quinta classe, e última classe não houve tendenciosidade.

As funções Log-normal e Gamma apresentaram praticamente o mesmo comportamento, com subestimativa das primeiras e quinta classe diamétrica, e, superestimaram a última classe.

\section{Discussão}

Considerando a tendência de distribuição apresentada pelos dados (Tabela 2), tem-se as classes centrais (Classes 3 e 4) apresentando maior concentração $(58,59 \%)$ de indivíduos. Mesmo com essa superioridade de representação dessas classes e a avaliação estatística descritiva (Tabela 3) demonstrando valores próximos para média, mediana e moda, a amostra não apresentou significância para distribuição normal.

Esse comportamento da variável diâmetro à altura do peito, segundo Jesus et al. (2017), caracteriza uma distribuição unimodal, representado por um povoamento em que não houveram intervenções silviculturais entre as classes diamétricas.

A curva da distribuição diamétrica possui assimetria negativa, isso demonstra que se tem mais indivíduos nas maiores classes, resultando na cauda do lado direito maior que a do lado esquerdo. Resultado diferente do observado por Araújo Júnior et al. (2013) em povoamentos de um híbrido de Eucalyptus grandis $e$ Eucalyptus urophylla com idades entre 2 e 5 anos, onde a cauda de distribuição foi considerada maior para o lado direito. Essa diferença pode estar relacionada a idade diferente entre os estudos, a origem, por se tratar de clone e não ter sofrido tratamento silvicultural.

A distribuição diamétrica apresentou curva leptocúrtica, devido ter apresentado uma medida de curtose maior que a da distribuição normal, resultado também observado por Machado et al. (2006). Essa característica que ocorre quando há maior quantidade de indivíduos com diâmetros próximos ao diâmetro médio do povoamento, elevando-se o pico da distribuição a um grau maior que em uma distribuição normal.

Em relação ao desempenho da função Weibull, com destaque para a função com três parâmetros, para caracterização da distribuição diamétrica, muitos são os estudos que pesquisadores obtiveram bons resultados ajustando essa função (Abreu et al. 2002; Binoti et al. 2010; Miguel et al. 2010; Binoti et al. 2015).

O estudo de Abreu et al. (2002) em povoamentos de Eucalyptus grandis situados no município de Luiz Antonio, estado de São Paulo, ajustando as funções probabilísticas Beta, Weibull 3P e $\mathrm{Sb}$ de Johnson, concluíram que a função Weibull, ajustada pelo método dos momentos, se mostrou mais precisa, considerando os resultados do teste de Kolmogorov-Smirnov.

Binoti et al. (2010) ao estudar um povoamento desbastado de um clone híbrido de eucalipto (Eucalyptus grandis $x$ Eucalyptus urophylla), localizado na região
Nordeste do Estado da Bahia, verificaram que todos os ajustes da função Weibull resultaram em aderência pelo teste de Kolmogorov-Smirnorv ( $p>0,01)$, comprovando a flexibilidade dessa função.

Téo et al. (2012), em estudo com Pinus taeda na região de Caçador-SC, relataram que as funções $\mathrm{Sb}$ de Johnson e Weibull 3P apresentaram os melhores resultados na representação da dinâmica da distribuição diamétrica e evidencia a flexibilidade da função em várias espécies.

Binoti et al. (2015) afirmam que, entre outras características, a boa correlação apresentada pelos parâmetros da Weibull 3P com características do povoamento tem sido fundamental à utilização dessa função.

Marangon et al. (2016) ao testarem funções de densidade de probabilidade para de espécies lenhosas da caatinga, semiárido pernambucano, verificaram que a função de Weibull 3P, foi a que melhor descreveu a distribuição diamétrica de Mimosa ophthalmocentra e Manihot carthaginensis, além de melhor descrever também o conjunto total de espécies.

De maneira geral, a explicação para a superioridade dessa função está no fato de que floresta equiâneas tendem a apresentar distribuições assimétricas à direita, em razão das árvores maiores apresentarem maiores taxas de crescimento do que as menores, gerando condições ideias para o ajuste dessa função, comprovando os resultados encontrado por Eisfeld et al. (2005).

Em relação a função que obteve pior ajuste, Téo et al. (2012) também obtiveram os piores resultados no ajuste da função Log-normal em todas classe de idade do estudo, que foram entre 5 e 8,9 anos em três classe de sítios. Essa função subestimou a última classe, que representa os diâmetros de maior interesse comercial para fins mais nobres, por exemplo, uso das toras para serraria.

A diferença entre as funções mostra a importância de se ajustar uma equação que explique a distribuição diamétrica do povoamento principalmente para quantificar os sortimentos das árvores. Além disso, Madi et al. (2017) evidencia a necessidade de selecionar o modelo mais preciso para cada caso analisado, sendo este um passo importante para a tomada de decisão do gestor florestal.

\section{Conclusões}

O modelo Weibull 3P obteve o melhor desempenho na modelagem da distribuição de diâmetro do povoamento do híbrido de Eucalytpus urophylla $x$ Eucalyptus grandis e segue distribuição leptocúrtica com assimetria à esquerda.

\section{Referências}

Abreu ECR, Scolforo JRS, De Oliveira AD, De Mello JM, Kanegae H (2002) Modelagem para prognose precoce do volume por classe diamétrica para Eucalyptus grandis. Scientia Forestalis/Forest Sciences, n. 61, p. 86-102.

Alvares CA, Stape JL, Sentelhas P C, De Moraes Gonçalves JL, Sparovek, G (2013) Köppen's climate classification map for Brazil. Meteorologische Zeitschrift, v. 22, n. 6, p. 711-728

Araújo Júnior CA, Leite HG, Castro RVO, Binoti DHB, Alcântara AEM, Binoti MLMS (2013) Modelagem da distribuição diamétrica de povoamentos de 
eucalipto utilizando a função Gama. Cerne [online]. 2013, vol.19, n.2, pp.307-314. ISSN 0104-7760. doi: http://dx.doi.org/10.1590/S010477602013000200015

Arce JE (2004) Modelagem da estrutura de florestas clonais de Populus deltoides Marsh. através de distribuições diamétricas probabilísticas. Ciência Florestal, v. 14, n. 1, p. 149-164.

Arnold TW (2010) Uninformative Parameters and Model Selection Using Akaike's Information Criterion. Journal of Wildlife Management, v. 74, n. 6, p. 11751178.

Binoti DHB, Leite HG, Nogueira, GS. Silva MLM, Garcia SLR, Cruz JP (2010) Uso da função Weibull de três parâmetros em um modelo de distribuição diamétrica para plantios de eucalipto submetidos a desbaste. Rev. Árvore [online]. 2010, vol.34, n.1, pp.147-156. ISSN 0100-6762. doi: http://dx.doi.org/10.1590/S010067622010000100016

Binoti DHB, Binoti MLMS, Leite HG, Melido RCN, Santos FLD (2012) Descrição da distribuição diamétrica de sistemas agrossilvipastoris utilizando as funções Weibull e hiperbólica. Revista Árvore, v. 36, n. 2 , p. $349-356$.

Binoti DHB, Binoti MLMS, Leite HG (2015) Modelagem da distribuição diamétrica de povoamentos equiâneos de eucalipto utilizando a função logística generalizada. Rev. Árvore [online]. 2015, vol.39, n.4, pp.707-711. ISSN 0100-6762. doi: http://dx.doi.org/10.1590/0100-67622015000400013

Eisfeld RL, Sanquetta CR, Arce JE, Maestri R, Weber KS (2005) Modelagem do Crescimento e da Produção de Pinus taeda L. por meio de função probabilística. Floresta, v. 35, p. 317-328.

Faria JC, Jelihovschi EG, Allaman IB (2017) fdth: Frequency Distribution Tables, Histograms and Polygons. UESC, Bahia, Brasil.

IBÁ. Relatório anual do setor brasileiro de árvores plantadas (2017) Indústria Brasileira de Árvores, Brasília p. 80.

IBGE. Instituto Brasileiro de Geografia de Estatística. Mapa do Clima do Brasil (2012) Disponível em: $<\mathrm{ftp}$ //geoftp.ibge.gov.br/informacoes_ambientais/cli matologia/mapas/brasil/clima.pdf>. Acesso em: 27 março de 2018

Jesus CM, Miguel EP, Rezende AV, Gaspar RO, Gatto A,Valadão MBX, Carrijo JVN (2017) Funções de densidade de probabilidade para estimativa das distribuições de variáveis dendrométricas em um povoamento clonal de eucalipto. Espacios, v. 38, p. 30.

Longue Júnior D, Colodette JL (2013) Importância e versatilidade da madeira de eucalipto para a indústria de base florestal. Pesquisa Florestal Brasileira, v. 33, n. 76, p. 429-438. doi https://doi.org/10.4336/2013.pfb.33.76.528

Machado SA, Santos AAP, Nascimento RGM, Augustynczik ALD, Zamin NT (2010) Modelagem da distribuição diamétrica de quatro espécies de
Lauraceae em um fragmento de Floresta Ombrófila Mista. Revista Ciências Exatas e Naturais, v. 12, n. 1, p. $91-105$.

Machado, SDA, Bartoszeck ACDPS, Figueiredo Filho A, Oliveira EB (2006) Dinâmica da distribuição diamétrica de bracatingais na região metropolitana de Curitiba. Rev. Árvore [online]. 2006, vol.30, n.5, pp.759-768. ISSN 0100-6762. doi: http://dx.doi.org/10.1590/S010067622006000500009 .

Madi JPS., Vendruscolo DGS, Silva CA, Chaves e Carvalho MPL, Chaves e Carvalho SP (2017) Univariate models to represent the diametric distribution of thinned stand of Tectona grandis Linn.F. Advances in Forestry Science, v. 4, n. Table 1, p. 119-123.

Marangon GP, Ferreira RLC, Da Silva JAA, Schneider PR, Loureiro GH (2016) Modelagem da distribuição diamétrica de espécies lenhosas da caatinga, semiárido pernambucano. Ciência. Florestal. [online]. 2016, vol.26, n.3, pp.863-874. ISSN 01039954. doi: http://dx.doi.org/10.5902/1980509824214

Miguel EP, Machado SDA, Figueiredo Filho A, Arce JE (2010) Using the Weibull fuction for prognosis of yield by diameter class in Eucalyptus urophylla stands. Cerne, v. 16, n. 1, p. 94-104. doi: http://dx.doi.org/10.1590/S010477602010000100011.

$\mathrm{R}$ development core team. R: A language and environment for statistical computing. R Foundation for Statistical Computing, Vienna, Austria. ISBN 3900051-07-0. Disponível em: <http://www.Rproject.org/>. Acesso: 22/03/2017.

Scolforo JRS (2006) Biometria florestal: modelos de crescimento e produção florestal. 1.ed. Lavras: UFLA/FAEPE, 393p

Téo SJ, Rocha SP, Bortoncello AC, Paz RA, Costa RH (2011) Dinâmica da distribuição diamétrica de povoamentos de Pinus taeda, na região de Caçador, SC. Pesquisa Florestal Brasileira, v. 2011, n. 67, p. 183-192.

Téo SJ, Bianchi JC, Peloso A, Nava PR, Marcon A, Ehlrs T, Costa RH (2012) Desempenho de funções de densidade probabilísticas para descrever a distribuição diamétrica de Pinus taeda, na região de Caçador, SC. Floresta, v. 42, n. 4, p. 741-754. doi: http://dx.doi.org/10.5380/rf.v42i4.25976

Venables WN, Ripley BD (2002). Modern Applied Statistics with S. 4th Edition. New York: Springer . ISBN 0-387-95457-0. 